\title{
Solution of Potential Problems Using an Overdetermined Complex Boundary Integral Method
}

\author{
W. W. SChULTZ and S. W. HoNG \\ Department of Mechanical Engineering and Applied Mechanics, \\ University of Michigan, Ann Arbor, Michigan 48109
}

Received February 17, 1988; revised October 31, 1988

\begin{abstract}
The advantages of solving potential problems using an overdetermined boundary integral element method are examined. Representing a 2-dimensional potential solution by an analytic complex function forms two algebraic systems from the real and imaginary parts of the discretized form of the Cauchy theorem. Depending on which boundary condition is prescribed, the real or the imaginary algebraic system is diagonally dominant. Computations show that the errors of the strong system (diagonally dominant) often have almost the same value as those of weak system (diagonally non-dominant) but with the opposite sign. The overdetermined system, composed of the combination of the real and imaginary parts, tends to average these errors, especially for circular contours. An error analysis and convergence studies for several geometries and boundary conditions are performed. A methodology for handling computational difficulties with contour corners is outlined. A further modification is proposed and tested that shows exponential convergence for circular contours. 1989 Academic Press, Inc.
\end{abstract}

\section{INTRODUCTION}

The boundary integral method (also called the panel method) is powerful for solving potential problems. The computational domain becomes the enclosing boundary, reducing the effective dimension of the problem by one. This significantly decreases the computational effort unless the contour is highly contored.

Boundary integral methods are usually derived from Green's theorem with an appropriate free-space Green's function. The solutions of 2-dimensional potential problems discussed here can be described by a complex analytic function. The boundary integral method can then be formulated from Cauchy's integral theorem (Greenhow, Vinje, Brevig, and Taylor [1], Lai and Hromadka [2]), resulting in two real algebraic systems. The elegance and simplicity of complex analysis carries over to computations as well. While the Green's function formulation and the complex method are not directly compared here, Dold and Peregrine [3] indicate that the latter method (from [1]) is clearly superior. Schultz [4] shows that the error of the complex formulation can be further reduced by solving these two systems of equations in a least-square sense for nonlinear breaking wave simulations.

In general, an integral equation is solved by a numerical model that assumes the boundary is composed of piecewise-polynomial curves (panels) and the known and 
unknown boundary values are approximated as piecewise-continuous functions along the boundary. The truncation error in solving an integral equation is determined by the approximations of the boundary shape and the known and unknown function values on the boundary. A rather extensive error anlysis of the conventional boundary integral method for a 2-dimensional Neuann problem is given by Hess [5]. He shows that using parabolically shaped elements with linearly varying singularity improves the accuracy when the effects of local element curvature are greater than that of the function derivatives. Unlike the Green's function formulations, the complex method does not depend on the shape of the contour between nodes. Most complex variable boundary integral methods adopt piecewise-linear representations of the complex functions. This linear interpolation results in secondorder accurate integration and typically gives second-order accurate solutions for the boundary integral solution as well.

The accuracy of the numerical solution is affected by the contour shapes, types of boundary conditions, nodal spacings, and the possible singular behavior of the resulting solutions. Moran [6] shows poor convergence for a sharp-angled contour (such as an airfoil) even when the solution there is smooth. Singularities usually occur at an abrupt change in boundary conditions or at boundary corners [12]. The solution accuracy then further degrades unless special precautions are taken such as supplementing the piecewise-linear representations with singular functions [7].

In this paper, the error is analyzed for the complex variable boundary integral method using elements with linearly varying functions for several cases. The advantages of solving both sets (real and imaginary) of algebraic equations together in a least-square sense are discussed. The integration error analyses show the correlation between solution error and factors such as geometric curvature, nodal spacing, and local solution gradients. We also present further modifications in Section 6 that show exponential convergence for smooth contours.

\section{Cauchy Boundary Integral formulation}

Cauchy's theorem is used to derive an integral equation in terms of the complex potential. The complex potential, $\beta$, is analytic inside the computational domain, $R$, and is given by

$$
\beta(z)=\phi+i \psi,
$$

where $\phi$ and $\psi$ are real functions that can be identified as the velocity potential and stream function, respectively, for ideal fluid flow problems. The 2-dimensional spatial representation is given by $z=x+i y$.

Figure 1 shows the problem domain and bounding surfaces. Cauchy's theorem gives

$$
\oint_{\partial R} \frac{\beta(z)}{z-\zeta_{k}} d z=i \alpha \beta\left(\zeta_{k}\right)
$$




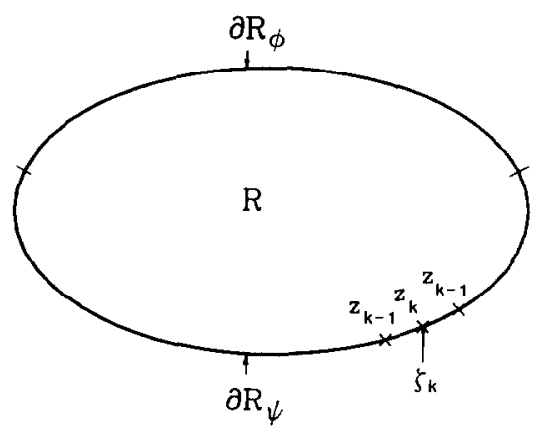

FIG. 1. Domain and bounding surfaces.

where $\alpha$ is 0 or $2 \pi$ if the location of the kernel singularity, $\zeta_{k}$, is outside or inside the boundary, respectively. If the kernel singularity is on the boundary $\left(\zeta_{k} \in \partial R\right), \alpha$ is equal to the included angle, and the integral is treated as principal-valued. For most of our computations, we take $\zeta_{k}$ to approach the boundary from the outside of the domain so that $\alpha$ is zero. The algebraic system is formed by discretization of the integral as explained in Section 3 and letting the kernel singularity approach each of the $N$ nodal points, $\zeta_{k} \rightarrow z_{k}$. Then a special limiting process is needed to evaluate the integration near $\zeta_{k}$.

The boundary contour $\partial R$ is composed of $\partial R_{\phi}$ and $\partial R_{\psi}$, where $\phi$ is given on $\partial R_{\phi}$ and $\psi$ is given on $\partial R_{\psi}$. For the problem to be well posed, either $\phi$ or $\psi$ must be specified along the entire boundary. This is related to the Dirichlet or Neumann problem, respectively, in the standard formulation. Although generalized (Robin) boundary conditions can be included in these computational schemes, we do not study them here. Hence, the boundary can be categorized as the following two types:

$$
\begin{array}{ll}
\operatorname{Re}\{\beta\}=\phi & \text { is given on } \partial R_{\phi} \\
\operatorname{Im}\{\beta\}=\psi & \text { is given on } \partial R_{\psi} .
\end{array}
$$

When the boundary condition is given exclusively as either $\phi$ or $\psi$ along the entire boundary, the solution is not unique since an additional real or imaginary constant can be added to the solution. This is related to a solvability condition for the Neumann problem. Therefore, an appropriate additional condition (or replacement condition if the problem is not to be overdetermined) is required. We have found that replacing the boundary condition at one point (assuming $\phi$ is known at every point except one, where $\psi$ is known) yields much less accurate results. Therefore the results we present here use an additional condition and hence all algebraic systems are overdetermined, although sometimes only by one extra equation.

To examine the corresponding singularity system in the standard formulation, the integrand in (2) can be rewritten as

$$
\frac{\phi e^{i \theta}}{z-\zeta_{k}} d s+i \frac{\psi e^{i \theta}}{z-\zeta_{k}} d s
$$


where $\theta$ is the tangent angle of the boundary with the positive $x$ axis. Hence, the contribution from the above differential element to the complex potential inside the domain is given by

$$
d \beta\left(\zeta_{k}\right)=\frac{1}{2 \pi}\left[\frac{\phi(-i) e^{i \theta}}{z-\zeta_{k}} d s+\frac{\psi e^{i \theta}}{z-\zeta_{k}} d s\right] .
$$

This relation shows that the integral equation (2) is equivalent to a system of distributed normal dipoles with strength $\phi$ and tangential dipoles of strength $\psi$ on the boundary. However, we can represent the potential field by using only one of the dipole systems, following the concepts of the indirect boundary integral method. (In the indirect method [16], the computational unknowns are the strengths of the distributed singularities, rather than the original solution as in the direct method.) Baker et al. [8] show that dipole distributions are more stable than source or vortex distributions for nonlinear breaking wave simulations. We could formulate a complex integral method similar to a combination of the sources and vortices by introducing, for example, a logarithmic singularity into (2) rather than the simple pole. Our preliminary numerical studies indicate that the pole in (2) performs better than other singularities, including higher order poles.

\section{Numerical Model}

Both the boundary contour and the complex potential are represented as piecewise-linear functions. Let $z_{j}$ and $\beta_{j}$ be the location and complex potential at the $j$ th node point. Our numerical model assumes $\beta$ on $j$ th panel can be described as

$$
\beta=\frac{1}{z_{j+1}-z_{j}}\left[\beta_{j}\left(z_{j+1}-z\right)+\beta_{j+1}\left(z-z_{j}\right)\right] .
$$

We can choose $\zeta_{k}$ of Eq. (2) at any point on the $k$ th panel. Although the panel midpoint and the node point are favored equally as $\zeta_{k}$ points, results where $\zeta_{k}$ is the node point are presented in this work. Our studies show no significant differences in accuracy and convergence rates between those two cases, hence we choose the endpoints since it is easier to code. We also have studied a more overconstrained problem by placing control points at the node points and the midpoints and have found no significant improvement. In Section 6 we examine the advantages of moving $\zeta_{k}$ away from the contour.

Letting $\zeta_{k}$ approach each node from the outside, yields the algebraic equivalent of (2) approximated as [1]

$$
\sum_{j=1}^{N} \beta_{j} \Gamma_{j k}=0 \quad \text { for } \quad k=1, \ldots, N,
$$


where

$$
\begin{aligned}
\Gamma_{j k} & =\frac{z_{j+1}-\zeta_{k}}{z_{j+1}-z_{j}} \ln \left(\frac{z_{j+1}-\zeta_{k}}{z_{j}-\zeta_{k}}\right)-\frac{z_{j-1}-\zeta_{k}}{z_{j}-z_{j-1}} \ln \left(\frac{z_{j}-\zeta_{k}}{z_{j-1}-\zeta_{k}}\right) \text { for } j \neq k, \\
\Gamma_{k k} & =\ln \left(\frac{z_{k+1}-z_{k}}{z_{k-1}-z_{k}}\right) .
\end{aligned}
$$

Equation (9) is evaluated using L'Hospital's rule when $j=k+1$ or $k-1$. Moving the unknown boundary conditions to the right-hand side gives the following complex algebraic system for unknown $\phi_{j}$ or $\psi_{j}$ :

$\sum_{j \in \partial R_{\psi}} \Gamma_{j k} \phi_{j}+i \sum_{j \in \partial R_{\phi}} \Gamma_{j k} \psi_{j}=-\sum_{j \in \partial R_{\phi}} \Gamma_{j k} \phi_{j}-i \sum_{j \in \partial R_{\psi}} \Gamma_{j k} \psi_{j} \quad$ for $\quad k=1, \ldots, N$.

$\Gamma_{j k}$ represents the influence of the $k$ th node kernel singularity on the $j$ th node complex potential $\beta_{j}$. In general, $\Gamma_{k-1, k}$, and $\Gamma_{k+1, k}$ have the dominant influence on $k$ th equation except for contours that are not convex or have sharp cusps. The imaginary part of $\Gamma_{k k}$ is nearly $-\pi$ and the real part is nearly zero for uniformly spaced nodes on smooth parts of the contour, and exactly $-\pi$ and zero, respectively, for flat contours. On the other hand, the imaginary parts of $\Gamma_{k-1, k}$ and $\Gamma_{k+1, k}$ are nearly zero while the real parts are not small.

Choosing the imaginary parts of system (11) for the $k$ th equation, where $\psi$ is given and the real parts where $\phi$ is given, results in a system matrix with a strong diagonal. Taking the other systems results in a system matrix with a weak diagonal. We now refer to the former system as the strong system and the latter system as the weak system. The strong system is very similar to a Fredholm integral equation of the second kind and the weak system is similar to a Fredholm integral equation of the first kind. As indicated in the introduction, we solve the weak and strong systems together as an overdetermined system in a least-squares sense and compare these results to those obtained from the individual systems.

\section{Truncation ERror in Numerical INTEgration}

The truncation error in the numerical integration is analyzed for the case of uniformly spaced nodes. In general, the dominant truncation error in (2) for the $k$ th control point occurs in the integration from $z=z_{k-1}$ to $z=z_{k+1}$, as shown in Fig. 1. We rewrite this part of the integral (2) as

$$
I_{k}=\int_{z_{k-1}}^{z_{k+1}} \frac{\beta(z)}{z-\zeta_{k}} d z
$$

For uniformly spaced nodes with slowly varying geometric curvature, we can examine the integral $I_{k}$ by expanding $\beta(z)$ at $z=\zeta_{k}$ as

$$
\beta(z)=\beta\left(\zeta_{k}\right)+\beta^{\prime}\left(\zeta_{k}\right)\left(z-\zeta_{k}\right)+\frac{1}{2} \beta^{\prime \prime}\left(\zeta_{k}\right)\left(z-\zeta_{k}\right)^{2}+O\left(\left|z-\zeta_{k}\right|^{3}\right)
$$


Using (12), Eq. (11) can be written as

$$
I_{k}=I_{k}^{(0)}+I_{k}^{(1)}+I_{k}^{(2)}+O\left(\left|z-\zeta_{k}\right|^{3}\right)
$$

where

$$
\begin{aligned}
& I_{k}^{(0)}=\beta\left(\zeta_{k}\right) \ln \left(\frac{z_{k+1}-z_{k}}{z_{k-1}-z_{k}}\right), \\
& I_{k}^{(1)}=\beta^{\prime}\left(\zeta_{k}\right)\left(z_{k+1}-z_{k-1}\right),
\end{aligned}
$$

and

$$
I_{k}^{(2)}=\frac{1}{4} \beta^{\prime \prime}\left(\zeta_{k}\right)\left[\left(z_{k+1}-z_{k}\right)^{2}-\left(z_{k-1}-z_{k}\right)^{2}\right] .
$$

Since our numerical model assumes $\beta$ varies linearly between nodes, the dominant truncation error in the integration of $I_{k}$ is $I_{k}^{(2)}$. Since the error of the integration is dependent on the square of the nodal spacing, we would expect the convergence of the solution of the integral equation to be second order as well or $O\left(1 / N^{2}\right)$. It can easily be shown that this dominant error term is cancelled when the contour is straight for three consecutive, equally spaced nodes. Hence it will come as no surprise that the boundary integral equation can be solved more easily when the contour is flat (or at least smooth) and the nodal spacing is uniform. We can also use the information on the dominant truncation error to improve the solution at corners (see Section 5.5).

For the special case of equally spaced nodes on a circular contour, i.e., $z_{k}=\exp (2 \pi i k / N)$, the dominant error becomes

$$
I_{k}^{(2)}=\frac{i}{2} \beta^{\prime \prime}\left(\zeta_{k}\right)\left[\sin \frac{4 \pi}{N}-2 \sin \frac{2 \pi}{N}\right]=O\left(1 / N^{3}\right) .
$$

This indicates that the most dominant error term for this case comes outside the range of integration of (11). For this special case, we will show from numerical experiments that handling the boundary integral equation in a special way will change the expected second-order convergence to fourth order.

When $\beta$ is linear, the integration is exact and the integral equation algorithm gives exact solutions. In Fig. 2, a very strong correlation between $\operatorname{Re}\left\{I_{k}^{(2)}\right\}$ and the solution error $\left(\psi_{\text {comp }}-\psi_{\text {exact }}\right)$ is shown for a solution domain inside a circular contour with uniformly spaced nodes when $\phi$ is given $\operatorname{as} \operatorname{Re}\{\sin z\}$. The correlations are nearly 1.0 and -1.0 for the weak and strong systems, respectively. Figure $3 \mathrm{a}$ shows that the strong and weak systems give approximately the same errors but of opposite sign. The error distribution along the same contour for another test function, in which $\beta=e^{z}$, is shown in Fig. $3 \mathrm{~b}$.

The results for a problem when the solution domain is outside the unit circle is given in Fig. 3c. This example represents a dipole in a uniform potential gradient. The imaginary part of the potential should be zero on the unit circle. To solve this problem, the uniform part must be subtracted so that there is no contribution from 


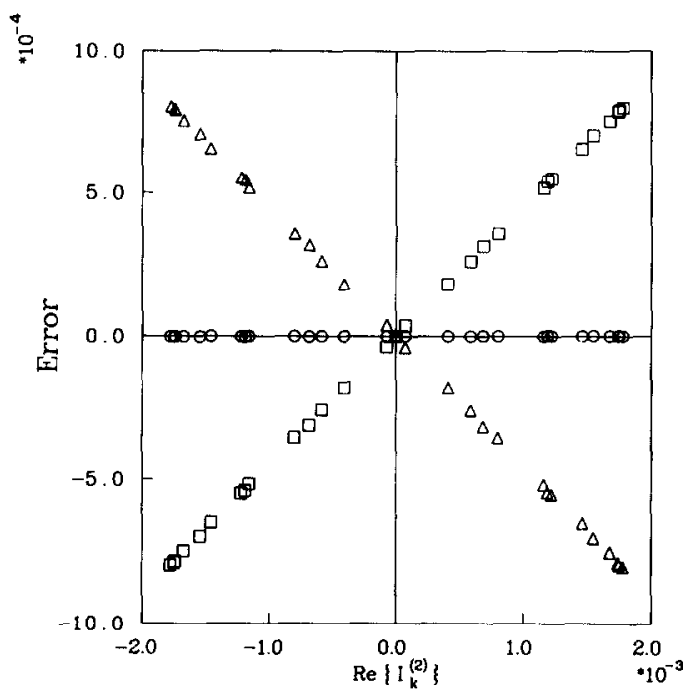

Fig. 2. Correlations between error and $\operatorname{Re}\left\{I_{k}^{(2)}\right\}$ (circular contour, inner problem, $\beta=\sin (z), \phi$ is given): squares--weak system; triangles-strong system; circles-overdetermined system.

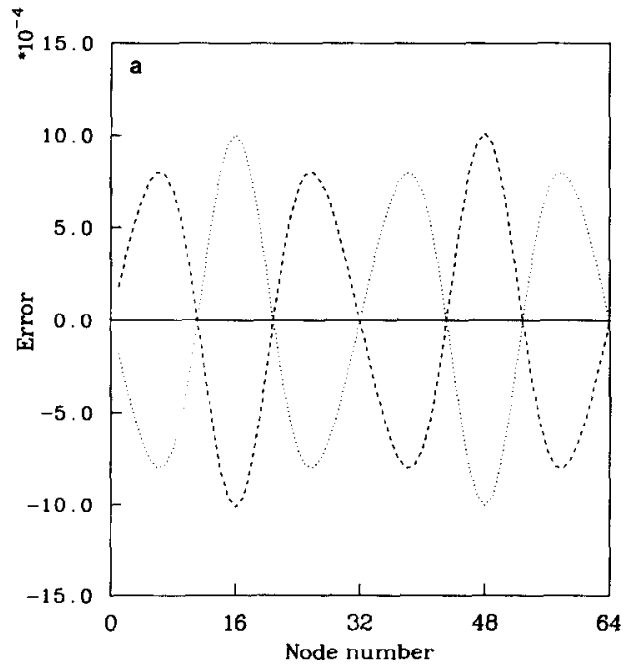

Fig. 3. Error distributions for uniform spacing on circular contours: dotted line-strong system; dashed line-weak system (overdetermined system errors not shown because they are too small at this scale). (a) Inner problem, $\beta=\sin (z), \phi$ is given; (b) Inner problem, $\beta=\exp (z), \phi$ is given; (c) Outer problem, $\beta=z+1 / z, \phi$ is given. 

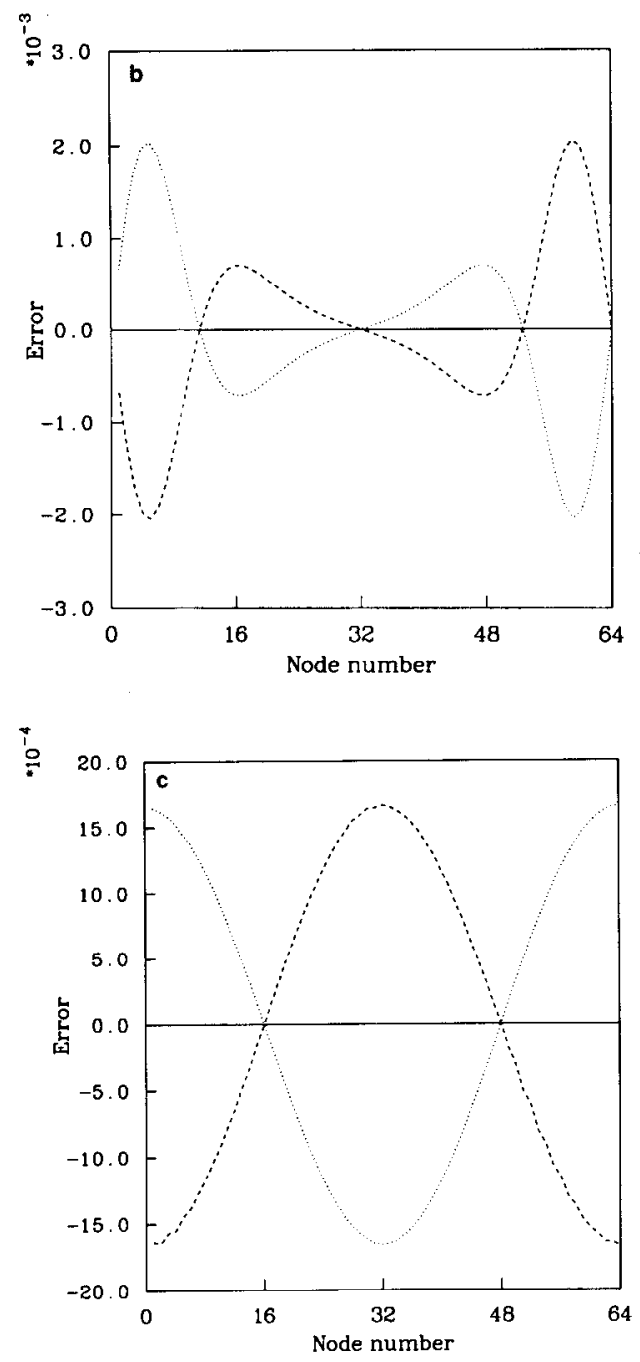

FIG. 3-Continued

the contour at infinity. A branch cut is chosen to connect the contour at infinity to the circular contour, which is then integrated in a clockwise sense. All examples in Fig. 3 show the typical error cancellation characteristics of the overdetermined system. The overdetermined system averages the errors of weak and strong systems so that the error distribution curves of the overdetermined system are nearly collapsed to the abscissa and hence not plotted. Other calculations show that this error cancellation for the outer problem is nearly complete when the dipole is moved from the origin but still well inside the contour.

It is noteworthy that the solutions of the weak system sometimes show small 
oscillations with wavelengths twice the nodal spacing, as shown in Fig. 3c. This common zig-zag instability is due in part to the nearly singular matrix. At times, when this numerical instability becomes large, the weak system matrix becomes so numerically singular that direct inversion is no longer possible. Then iterative refinement is required. As described in the next section, we use an iterative procedure to solve the algebraic system. When the zig-zag instability becomes quite large, the number of iterations increases significantly. Often improved solutions (with
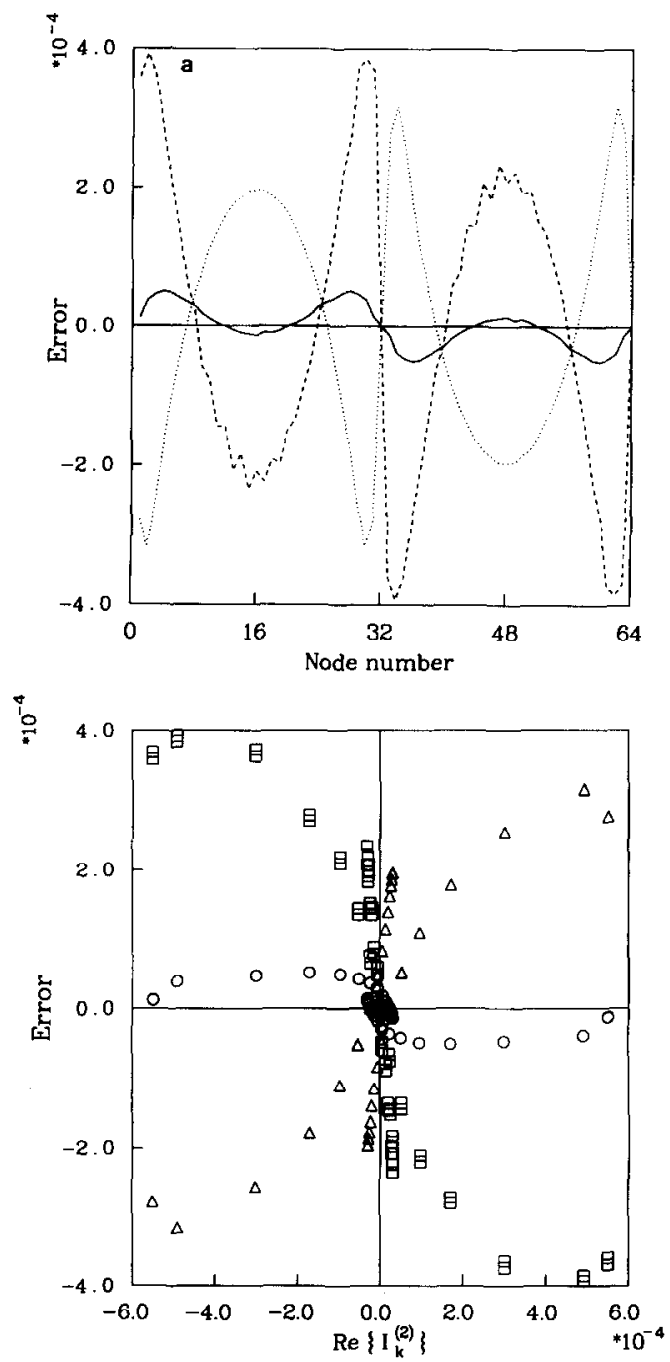

FIG. 4. Error for elliptical contours ( $e=0.9165$, inner problem, $\beta=\sin (z), \phi$ is given); (a) Error distribution: dotted line-strong system; dashed line-weak system; solid line-overdetermined system. (b) Corrleations between error and $\operatorname{Re}\left\{I_{k}^{(2)}\right\}$ : squares-weak system; triangles-strong system; circlesoverdetermined system. 
TABLE I

Correlation Number between Error and $\operatorname{Re}\left(I_{k}^{(2)}\right)$

\begin{tabular}{llll}
\hline Eccentricity & Weak & Strong & Overdetermined \\
\hline 0.0 & 0.9997 & -0.9986 & 0.1915 \\
0.6 & 0.9745 & -0.9711 & 0.3292 \\
0.8 & 0.9075 & -0.8845 & 0.5856 \\
0.9165 & 0.8367 & -0.7974 & 0.6133 \\
0.9798 & 0.4809 & -0.7767 & 0.6451 \\
\hline
\end{tabular}

Note. Ellipse, inner problem, $\beta=\sin (z), \phi$ given.

smaller oscillations) are achieved with fewer iterations (less rigorous convergence criteria). This behavior was never seen for the strong or overdetermined systems.

For noncircular contours, the errors contributed from off-diagonal elements grow as the local geometric curvature increases. Figure 4 shows the error distribution curve and the correlation between error and $\operatorname{Re}\left\{I_{k}^{(2)}\right\}$ when $\phi$ is given for an elliptical contour with an eccentricity of 0.9165 . For the examples shown here the major axis of unit radius is centered on the $x$-axis, so that the contour is given by $x^{2}+y^{2} / b^{2}=1$, where $b$ is the minor axis given by $b^{2}=1-e^{2}$.

A relatively strong correlation still exists, but the error of the weak system is more sensitive near $\operatorname{Re}\left\{I_{k}^{(2)}\right\}=0$. In this case, the cancellation characteristic of the overdetermined system is no longer nearly perfect, as shown in Fig. 3. Table I shows the correlation numbers between the solution error and $\operatorname{Re}\left\{I_{k}^{(2)}\right\}$ for various ellipses with eccentricities of 0.0 to 0.9798 (minor axes in the $y$ direction of 1 to 0.1 ). The weak system loses its strong correlation at an eccentricity of 0.9798 , where large spurious oscillatory behavior develops in the solution.

We will show that the error cancellation of the overdetermined system seems more dependent on the variation of geometric curvature and on the nodal spacing than on the solution characteristics.

\section{Numerical INVESTIGATION}

Comparisons of accuracy, convergence rate, and computing time are made for weak, strong, and overdetermined sets of system (11). Effects of nonuniform nodal spacing, types of boundary conditions, nearly singular solution characteristics, and contour shape on the solution errors are investigated.

The overdetermined matrix problem can be solved two ways. One method uses a routine based on the Householder transformation [9]. This routine takes an order of $U^{2} V$ operations, where $U$ is the number of unknowns and $V$ is the number of equations. The other uses a conjugate gradient iterative technique [10], which solves the problem in an order of $U V L$ operations, where $L$ is the number of itera- 
tions. The computational savings of the iterative technique would be important if time marching were desired, especially since a good initial guess is available from the previous time step. However, even with a homogeneous initial guess, a typical solution for a nonsingular matrix requires less than 10 iterations.

The iterative solver often takes more computing time than the Householder transformation method for the weak system, but the Householders method is inaccurate because the system matrix in this case is nearly singular. Iterative methods are more reliable when the system matrix is nearly singular. Here, we use the conjugate gradient iterative method with a zero initial iterate to compare the efficiency of the three systems.

\subsection{Rate of Convergence and Computing Time}

The root mean square errors $\left(E_{2}\right)$ and corresponding computing times are examined as a function of the number of nodes to determine convergence rates and computational efficiency. The maximum errors $\left(E_{\infty}\right)$ follow a very similar pattern and hence are not shown. Figure 5 shows $E_{2}$ for the three systems in $\log -\log$ scale for uniformly spaced nodes on a circular contour when $\phi$ is given as $\operatorname{Re}\{\sin z\}$. Single precision (seven digits) results are limited to approximately five decimal point accuracy as roundoff error dominates truncation error beyond $N=32$. The overdetermined system is more accurate by one digit at $N=16$, and the convergence rate of the overdetermined system is twice that of the weak or strong systems.

In Fig. 6, single precision computing times for an Apollo 4000 workstation are shown. For the same $N$, the computing time of the overdetermined system is the

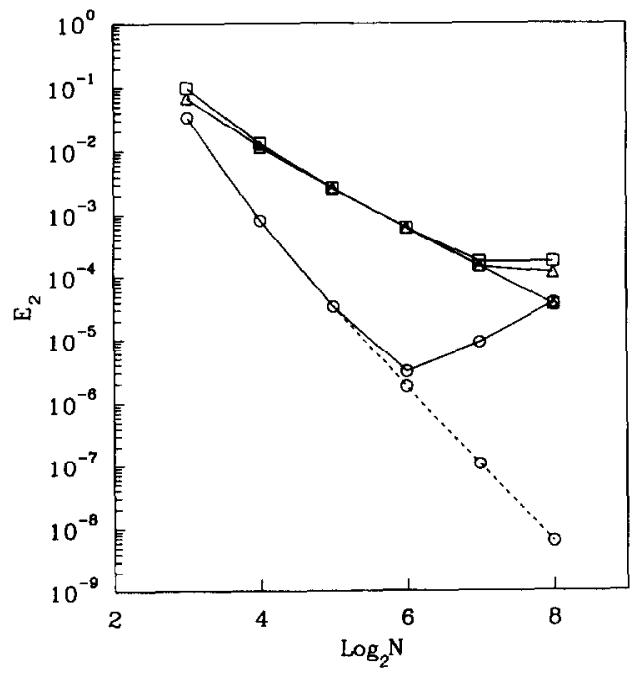

Fig. 5. Truncation error convergence for circular contours (inner problem, $\beta=\sin (z), \phi$ is given): squares-weak system; triangles-strong system; circles-overdetermined system; dotted line-double precision; dashed line - single precision. 


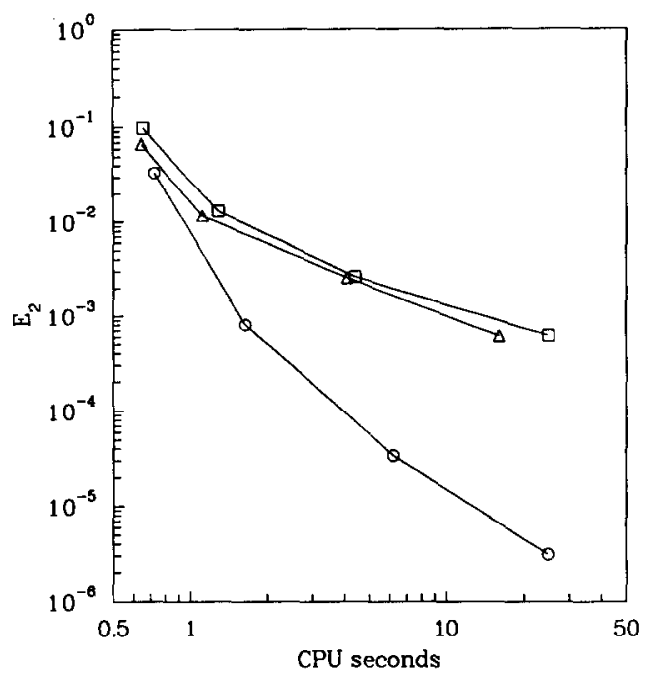

Fig. 6. Relation between $E_{2}$ errors and computing times (circular contour, inner problem, $\beta=\sin (z)$, $\phi$ is given): squares-weak system; triangles-strong system; circles-overdetermined system.

largest although not by a factor of 2 as when using a direct solver. The computing time to solve the weak system increases rather rapidly when $N>32$ as the system matrix becomes more singular and the number of iterations increases. Figure 6 clearly shows that the overdetermined solution is the most efficient of the three systems for this example.

The effect of the contour shape on the efficiency of inner problems is examined for elliptical contours with various eccentricities $(e=0.0$ to 0.995$)$ for the same $\beta$.

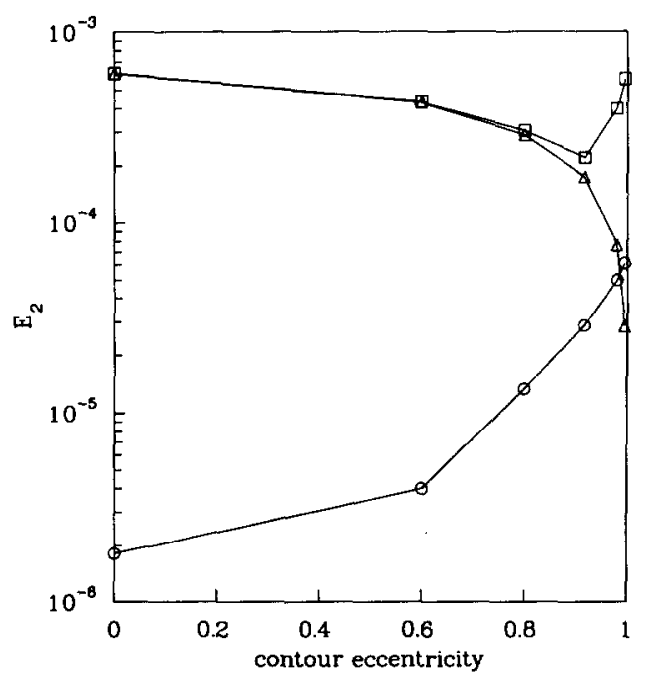

FIG. 7. $E_{2}$ errors for various elliptical contours $(N=64$, inner problem, $\beta=\sin (z), \phi$ is given): squares-weak system; triangles-strong system; circles-overdetermined system. 
The diameter of the major axis is fixed to be 2.0 and centered on the $x$ axis. As the eccentricity increases, the $E_{2}$ of the overdetermined system increase, but those of the strong systems decrease, as shown in Fig. 7. The $E_{2}$ of the weak system for $e \geqslant 0.9165$ increase due to the more oscillatory behavior of the solution. In Table II, the $E_{2}$ are given as a function of number of nodes for various ellipses. In most cases, the overdetermined system gives the best results, except for highly eccentric elliptical contours where the weak system's oscillations affect the overdetermined solution as well.

The $E_{2}$ convergence rates determined at the largest computed $N$ are compared in Table II for inner problems for various eccentricity. Astonishingly, the convergence rate of the overdetermined system for a circular contour $(e=0)$ approaches 4.0 . The convergence rates of all the other examples are the predicted 2.0.

$E_{2}$ for outer problems, when $\beta=1 / z$, are also calculated and given in Table IIl for various ellipses $(0 \leqslant e \leqslant 0.995)$ with a double precision program. The results of

TABLE II

$E_{2}$ Convergence Rates for Inner Problem

\begin{tabular}{|c|c|c|c|c|c|c|}
\hline \multirow[b]{2}{*}{$e$} & \multirow[b]{2}{*}{ System } & \multicolumn{4}{|c|}{$-\mathrm{LOG}_{10} E_{2}$} & \multirow{2}{*}{$\begin{array}{l}\text { Approx. } \\
\text { Conv. rate }\end{array}$} \\
\hline & & $N=32$ & $N=64$ & $N=128$ & $N=256$ & \\
\hline \multirow[t]{3}{*}{0.0} & Weak & 2.578 & 3.212 & 3.829 & 4.437 & 2.022 \\
\hline & Strong & 2.588 & 3.215 & 3.829 & 4.438 & 2.021 \\
\hline & Overdet. & 4.470 & 5.743 & 6.979 & 8.198 & 4.051 \\
\hline \multirow[t]{3}{*}{0.6} & Weak & 2.732 & 3.366 & 3.982 & 4.590 & 2.022 \\
\hline & Strong & 2.743 & 3.370 & 3.984 & 4.592 & 2.020 \\
\hline & Overdet. & 4.646 & 5.399 & 5.997 & 6.598 & 1.993 \\
\hline \multirow[t]{3}{*}{0.8} & Weak & 2.880 & 3.518 & 4.135 & 4.744 & 2.022 \\
\hline & Strong & 2.911 & 3.541 & 4.156 & 4.765 & 2.022 \\
\hline & Overdet. & 4.286 & 4.874 & 5.470 & 6.071 & 1.999 \\
\hline \multirow[t]{3}{*}{0.9165} & Weak & 2.904 & 3.659 & 4.281 & 4.891 & 2.026 \\
\hline & Strong & 3.123 & 3.761 & 4.383 & 4.994 & 2.031 \\
\hline & Overdet. & 3.946 & 4.543 & 5.143 & 5.745 & 2.000 \\
\hline \multirow[t]{3}{*}{0.9798} & Weak & 2.642 & 3.403 & 4.373 & 5.037 & 2.206 \\
\hline & Strong & 3.591 & 4.122 & 4.727 & 5.357 & 2.093 \\
\hline & Overdet. & 3.619 & 4.305 & 4.919 & 5.521 & 1.999 \\
\hline \multirow[t]{3}{*}{0.995} & Weak & 2.684 & 3.249 & 4.104 & 4.737 & 2.103 \\
\hline & Strong & 3.926 & 4.547 & 5.092 & 5.630 & 1.805 \\
\hline & Overdet. & 3.578 & 4.215 & 4.864 & 5.489 & 2.079 \\
\hline
\end{tabular}

Note. Ellipse, $\beta=\sin (z), \phi$ given, even nodal spacing. 
TABLE III

$E_{2}$ Convergence Rates for Outer Problem

\begin{tabular}{|c|c|c|c|c|c|}
\hline \multirow[b]{2}{*}{$e$} & \multirow[b]{2}{*}{ System } & \multicolumn{3}{|c|}{$-\mathrm{LOG}_{10} E_{2}$} & \multirow{2}{*}{$\begin{array}{l}\text { Approx. } \\
\text { Conv. rate }\end{array}$} \\
\hline & & $N=32$ & $N=64$ & $N=128$ & \\
\hline \multirow[t]{3}{*}{0.0} & Weak & 2.3132 & 2.9299 & 3.5393 & 2.024 \\
\hline & Strong & 2.3162 & 2.9306 & 3.5395 & 2.023 \\
\hline & Overdet. & 4.7798 & 6.0105 & 7.2208 & 4.021 \\
\hline \multirow[t]{3}{*}{0.6} & Weak & 2.2921 & 2.9161 & 3.5288 & 2.035 \\
\hline & Strong & 2.1563 & 2.7702 & 3.3789 & 2.022 \\
\hline & Overdet. & 3.6176 & 4.2079 & 4.7974 & 1.958 \\
\hline \multirow[t]{3}{*}{0.8660} & Weak & 1.8871 & 2.5365 & 3.1584 & 2.066 \\
\hline & Strong & 1.8364 & 2.4573 & 3.0709 & 2.038 \\
\hline & Overdet. & 3.0694 & 3.7652 & 4.3682 & 2.003 \\
\hline \multirow[t]{3}{*}{0.9165} & Weak & 1.6468 & 2.3176 & 2.9447 & 2.083 \\
\hline & Strong & 1.6530 & 2.2783 & 2.8956 & 2.051 \\
\hline & Overdet. & 2.6897 & 3.5067 & 4.1247 & 2.053 \\
\hline \multirow[t]{3}{*}{0.9798} & Weak & 0.8131 & 1.5688 & 2.2367 & 2.219 \\
\hline & Strong & 0.9281 & 1.5898 & 2.2171 & 2.154 \\
\hline & Overdet. & 1.2885 & 2.6238 & 3.4247 & 2.661 \\
\hline \multirow[t]{3}{*}{0.9950} & Weak & 0.5428 & 0.6898 & 1.4458 & 2.511 \\
\hline & Strong & 0.1215 & 0.8045 & 1.4655 & 2.196 \\
\hline & Overdet. & 0.2512 & 1.1748 & 2.5264 & 4.490 \\
\hline
\end{tabular}

Note. Ellipse, $\beta=1 / z, \phi$ is given even nodal spacing.

the overdetermined system are the best for all cases tested. The convergence rates approach 2.0 for the weak and strong systems. The convergence rates of the overdetermined system also approach 2.0 except at $e=0$, which again exhibits fourth-order convergence. The apparent high convergence rate for the overdetermined system on highly eccentric ellipses returns to second order as $N \rightarrow \infty$. As the eccentricity increases, $E_{2}$ increases for all systems due to the more rapid variation of the function and the kernel.

Since our numerical model uses piecewise-linear representation for the potential function, we expect second-order convergence. However, for some cases, the convergence rates of the overdetermined system approach fourth order due to the cancellation of errors between the weak and the strong systems. Our examples show that these cancellations occur when the contour is circular (or nearly so) with uniformly spaced nodes. Some calculations not described here have shown third- and sixth-order convergence for the strong and overdetermined systems, respectively, when a cubic-spline representation is used for $\beta$. 


\subsection{Nonuniform Nodal Spacing}

Sometimes nonuniform nodal spacing is desirable when solution refinement is needed due to large solution gradients. Also, the nodal spacing in problems with convective nodes such as free surface flow problems becomes nonuniform. This can cause numerical instabilities, so that a filtering scheme or a regridding scheme to maintain uniform spacing is needed [11].

Figures $8 \mathrm{a}$ and $\mathrm{b}$ show the same example as in Fig. 3a, except that node 1 is
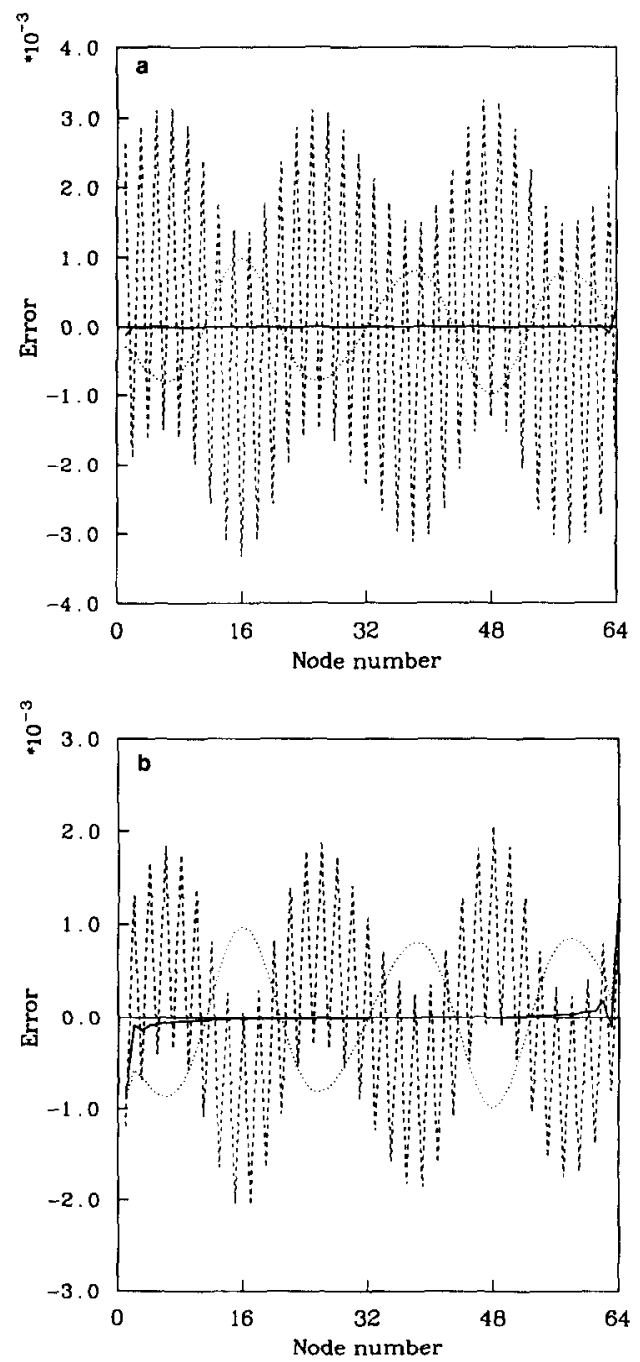

FIG. 8. Error distributions for nonuniformly spaced nodes (inner problem, $\beta=\sin (z), \phi$ is given): (a) Node 1 moved 0.1 of uniform spacing towards node 2. (b) Node 1 moved 0.5 of uniform spacing towards node 2 . 
moved towards node 2 by the stated fraction of the otherwise uniform spacing. In this case, the real part of $\Gamma_{k k}$ is no longer small. Moreover, the dominant off-diagonal terms $\Gamma_{k-1, k}$ and $\Gamma_{k+1, k}$ are also changed significantly while the imaginary part of $\Gamma_{k k}$ remains around $-\pi$. The results show that the strong and overdetermined system errors are affected only near that nonuniformly spaced node. As the nonuniformity increases, the solution of the weak system deteriorates globally due to the zig-zag instability. However, if a numerical filter were used to eliminate oscillations with wavelengths equal to the node spacing, the weak system error is comparable with the strong system and is again of opposite sign. This is why the solution for the overdetermincd system remains very good. Note that the error curves of the overdetermined system cannot be distinguished from the axis except near the moved node, especially when the nonuniformity is small as in Fig. 8a.

The correlation between $I_{k}^{(2)}$ and the solution error is still strong for strong systems, except around the $k$ th node, but it is very poor for the weak system. The computing time for the weak system increases rapidly as the nonuniformity increases, since the number of iterations grows rapidly as the matrix becomes more numerically singular.

It can be shown that the order of csystem has large reductions in error, especially when the nonuniformities are small.

\subsection{Mixed Boundary Condition Problems}

When the boundary contour is composed of both $\partial R_{\phi}$ and $\partial R_{\psi}$, the intersecting node can present a difficulty even without any geometric discontinuity. We could expect the solution to be singular at the intersecting node. We also would usually expect that the change in boundary conditions to occur at a corner in the boundary contour (which brings other difficulties that will be discussed in Section 5.5). We do not consider either case here. Either $\phi$ or $\psi$, or both can be treated as known at the intersecting node. When both are described, the method is often considered as "double nodes" in boundary integral methods, and is shown in some cases to give improved results [11].

We examine a circular contour with $\phi$ given on the upper half $(y>0)$ and $\psi$ on the lower half. Figure 9 shows the error distribution curves for two cases when $\beta=\sin (z)$. Only one boundary condition is prescribed at each node in Fig. 9a, while both $\phi$ and $\psi$ are given at the intersecting nodes in Fig. 9b. In either cases the error of the overdetermined system is much smaller while the weak system solution again shows oscillatory behavior. The error is reduced slightly by prescribing both boundary conditions to the nodes on the $x$-axis, i.e., nodes 1 and 33 .

$E_{2}$ for these two cases are compared in Fig. 10. The solutions when the additional known boundary constraints are used are better for all three systems. Convergence rates remain the same and the improvement in accuracy is small. There is also no large local error at the intersection point. We conclude that the change in boundary condition type by itself is not difficult to handle computationally. 

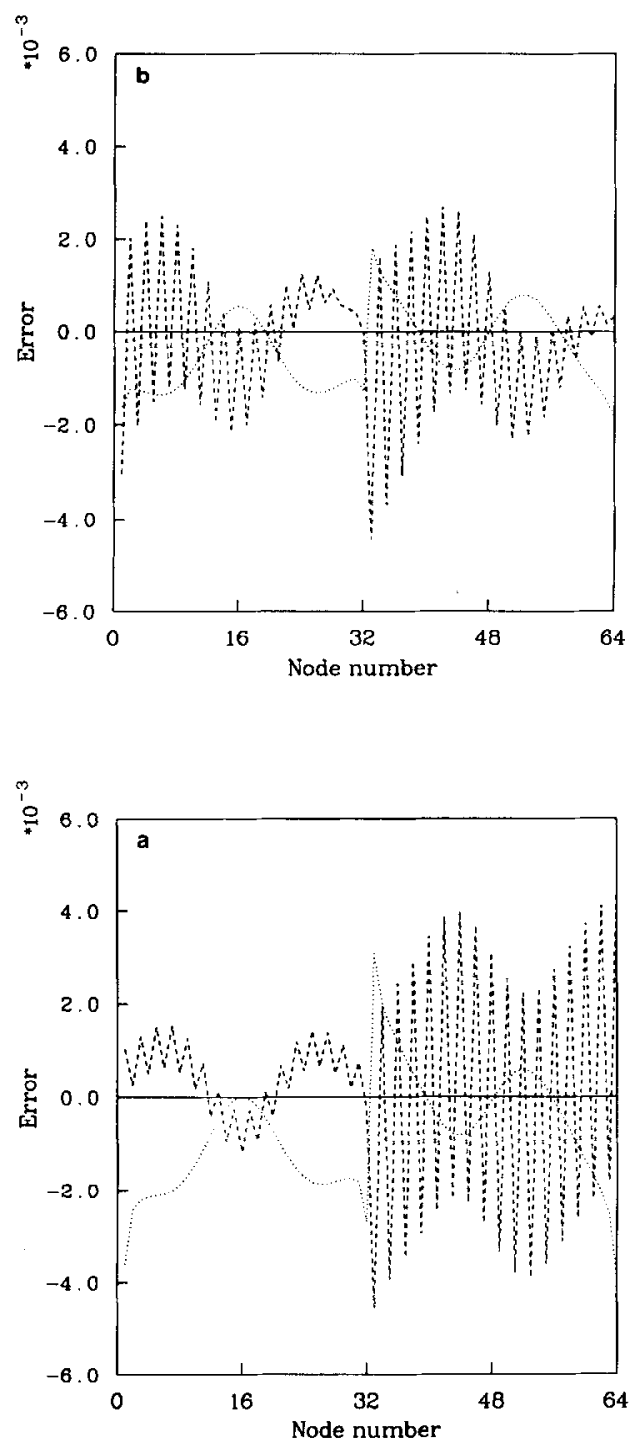

Fig. 9. Error distribution for a mixed boundary type problem (circular contour, $N=64$, inner problem, $\beta=\sin (z)$ ): dotted line-strong system; dashed line-weak system (overdetermined system errors not shown because they are too small at this scale). (a) Only one boundary condition prescribed on nodes 1 and 33. (b) Both $\phi$ and $\psi$ are given at the intersecting nodes 1 and 33 . 


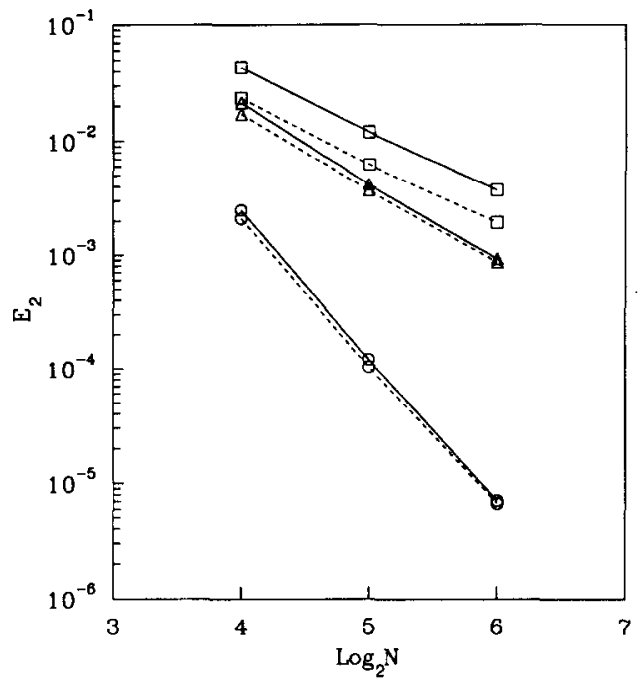

Fig. 10. Error convergence of mixed type problems (circular contour, inner problem, $\beta=e^{-i z}$ ): squares-weak system; triangles-strong system; circles-overdetermined system; dashed line-both conditions prescribed at intersecting nodes; solid line—one condition prescribed at intersecting nodes.

\subsection{Singularity Near Contour}

When the boundary condition values change abruptly due to nearby singularities, the solution error at nodes near those singularities is expected to increase. As an example, the error for a unit radius circular contour where the solution has a simple pole near the contour is examined, i.e., $\phi$ is given as the real part of

$$
\beta=\frac{1}{z-(1+\varepsilon)},
$$

where $\varepsilon$ is a small positive constant. The solution errors for $\psi$ of the strong system increase mainly at nodes near the singularity (near node 1), while those of the weak system exhibit oscillatory behavior peaking near the singularity, as shown in Fig. 11a. As $\varepsilon$ increases, the oscillation amplitude diminishes and the weak system finally recovers an error opposite to the strong system.

$E_{2}$ shown in Fig. 12 again show the advantage of the overdetermined system in accuracy and convergence rate. The convergence rate for the overdetermined system is again 4.0 and those of the other systems are 2.0. For this singular function, the nodal spacing must be less than $\varepsilon$ before the asymptotic convergence rate is achieved. We note that the weak system is unstable due to an abrupt change in $I_{k}^{(2)}$, which again implies that the matrix of the weak system is nearly singular. The oscillation amplitude of the weak system for this example was particularly dependent on the convergence criteria of the conjugate gradient iteration procedure. 

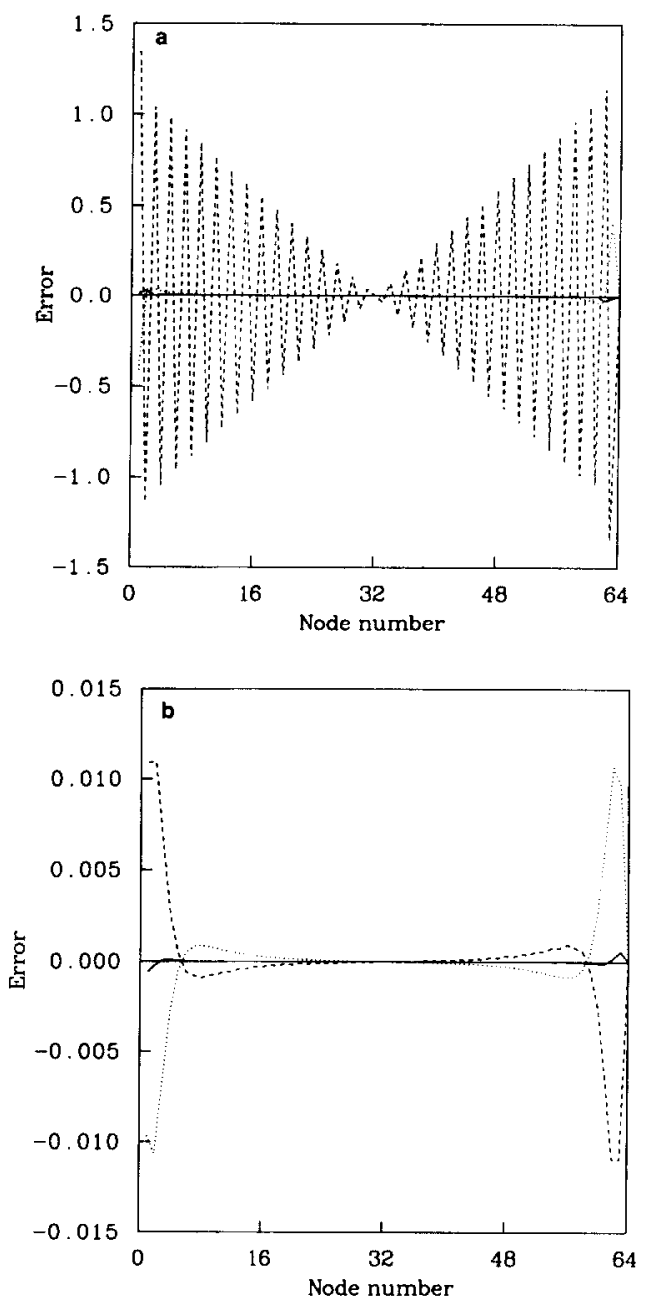

Fig. 11. Error distributions for singular function case (circular contour, inner problem, $\phi=\operatorname{Re}\{(1 /(z-(1+\varepsilon))\}$ is given): dotted line-strong system; dashed line-weak system; solid line-overdetermined system. (a) $\varepsilon=0.125$; (b) $\varepsilon=0.5$.

\subsection{Contours with Corners}

The boundary integral accuracy is known to deteriorate in the vicinity of contour corners due to large geometric curvature. Moreover, the solution is usually singular at corners. Grisvard [12] has the most mathematically complete discussion of numerical singularities arising from the non-smooth domain for elliptic boundary value problems. Pina, Fernandes, and Brebbia [13] suggest using mesh refinements to overcome the difficulties of nonoptimal convergence arising from an irregular 


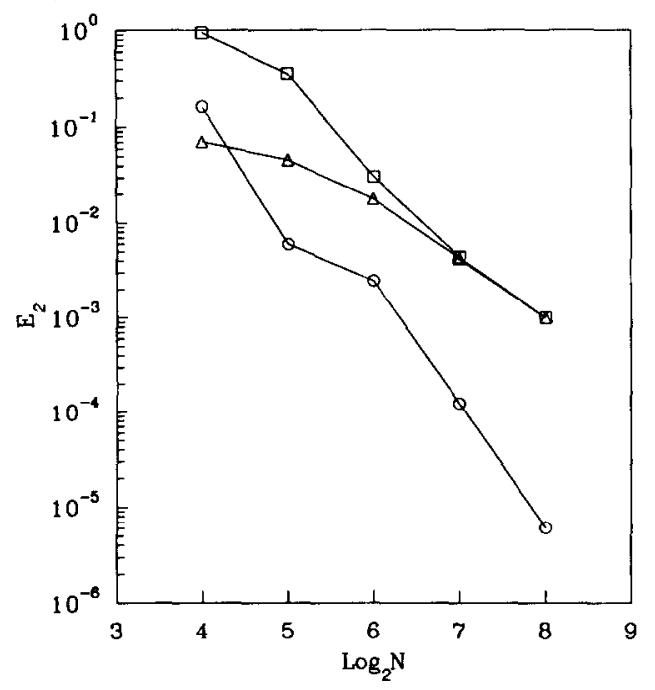

Fig. 12. $E_{2}$ errors for singular function case (circular contour, inner problem, $\phi$ is given, $\varepsilon=0.25$ ): dotted line-strong system; dashed line—weak system; solid line-overdetermined system.

boundary contour. If the solution is known to be singular or to vary rapidly at a corner, one can also try refined meshes (Lin, Newman, and Yue [14]) or a modified variable made by subtracting out the local singular solutions from the original variable (Kelmanson [7]) to enhance the accuracy. Mesh refinements near corner can create difficulties with numerical instability such as at the free surface of the wave maker problem [11].

Even if the solution is not singular at a corner, error is induced in large part by the contribution from the abrupt change of geometric curvature. This can be seen in Eq. (17) for $I_{k}^{(2)}$. At a corner, the term $\left(z_{k+1}-\zeta_{k}\right)^{2}-\left(z_{k-1}-\zeta_{k}\right)^{2}$ is not small. If $\beta^{\prime \prime}\left(\zeta_{k}\right)$ is nonzero at the corner, the error in numerical integration will be much larger there. In this case, the error has its maximum near the corner and can spread to the entire contour.

A modified circular contour with a corner (Fig. 13) is used to test the effects of the corner on the solution accuracy. The nodal spacing is adjusted along the straight segments of the contour to ensure the same number of nodes in each quadrant of the complex plane. In this case, the imaginary part of $\Gamma_{k k}$ at the corner stays at $-\pi / 2$ as the number of nodes increases. However, the off-diagonal influence coefficients are strongly affected by the corner and any refinement there.

As Fig. 14a shows, the error of the strong system is concentrated at the corner while staying at the previous error levels on the smooth part of the contour as shown in Fig. 3a. The error of the weak system solution oscillates over the entire contour. However, if a numerical filter were used to eliminate oscillations with wavelengths equal to the node spacing, the weak system would actually have a smaller error than the strong system. The computing time (the number of itera- 


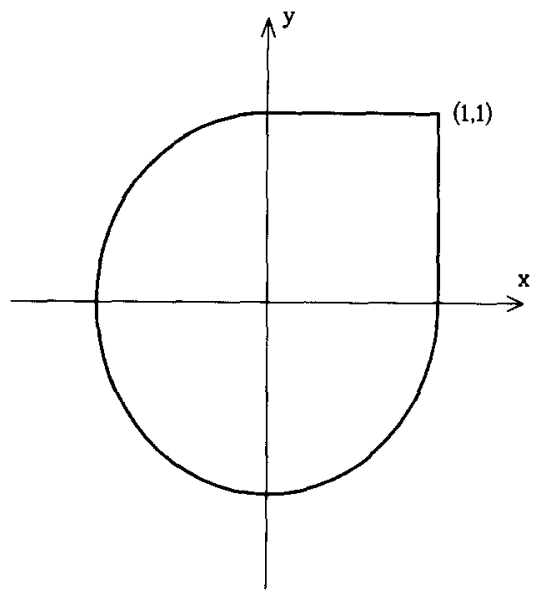

FIG. 13. Modified circular contour with a corner.

tions) to solve the weak system increases rapidly due to the corner. The solution of the overdetermined system is still significantly better, but the maximum error is one thousand times greater than the solution of the overdetermined system for the smooth contour shown in Fig. 3a. All three approaches show second-order convergence for contours with corners.

The given solution for this example is analytic at the corner, but we still have very grave computational difficulties there. To make the integration error smaller, we develop a modified complex potential to make $I_{k}^{(2)}=0$ at the corner by defining

$$
\beta_{0}(z)=\beta-\frac{1}{2}\left(z-\xi_{0}\right)^{2} \beta^{\prime \prime}\left(\xi_{0}\right),
$$

where $\xi_{0}$ is the coordinate of the corner. Thus, the second derivative of the modified complex potential $\beta_{0}$ is forced to be zero at the corner. This eliminates the highestorder error term in our piecewise-linear integration routine (Eq. (16)) at the corner.

Figure 14b shows improved results using this modification, especially at the corner. All six solutions (weak, strong, and overdetermined systems for both the modified and unmodified formulations) still appear to be second-order convergent for contours with corners. However, the $E_{2}$ or $E_{\infty}$ for fixed $N$ are significantly improved by the modified method. For this example, with $N=64$, these errors are reduced by a factor of 50 .

These results suggest an iterative method for more accurate solutions to nontest problems, when the unknown value of $\beta^{\prime \prime}\left(\xi_{c}\right)$ is updated to a more correct value. Further developments of this method are required for problems having more than one corner and for the outer problems. It is also expected that even more accurate solutions can be obtained by requiring the higher derivatives of the modified complex potential to be zero at corners. 

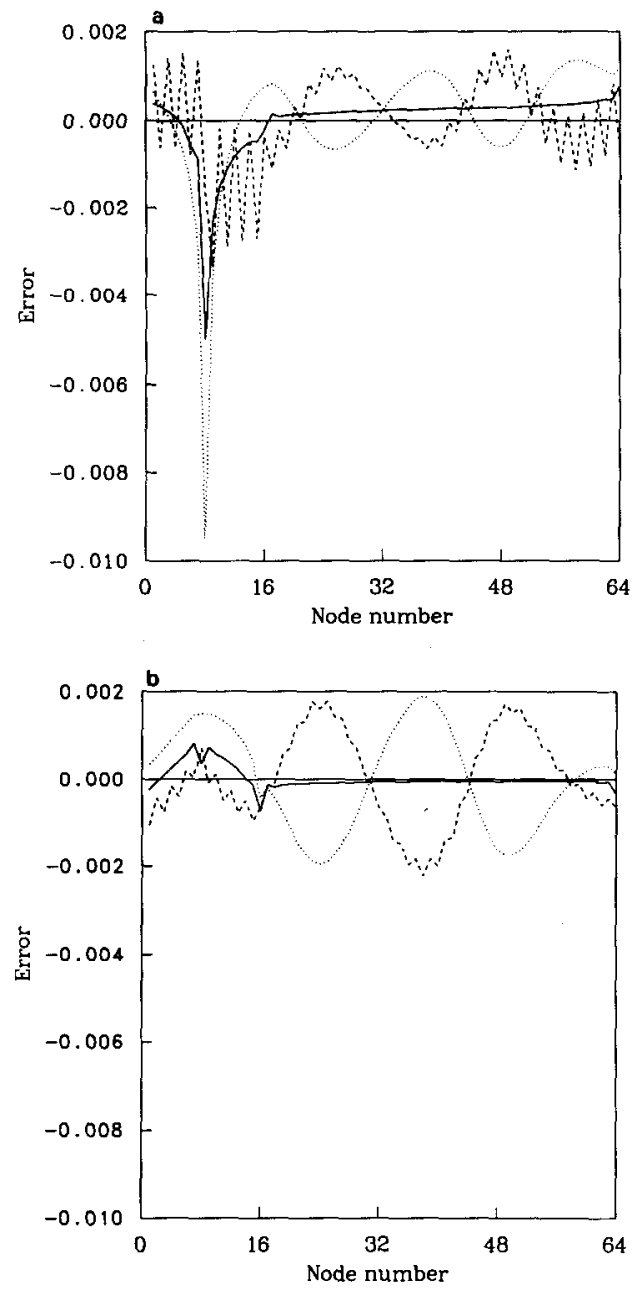

Fig. 14. Error distribution for a corner problem (inner problem, $\beta=\sin (z), \phi$ is given, corner node is 7): dotted line-strong system; dashed line-weak system; solid line-overdetermined system.

(a) Unmodified; (b) Modified.

\section{Desingularized Boundary Integral Method}

Patterson and Sheikh [15] introduce a regular boundary integral method by moving the singularity of the fundamental solution away from the boundary contour. Their rationale for doing this appears to be chiefly for desingularizing the kernel. Hence, all integrals can be computed numerically. They also claim the method "tolerates higher order singularities" and base these conclusions on comparisons with finite element methods for elastostatic problems. 
We show here that moving $z_{k}$ in Eq. (2) well outside the boundary has several advantages over the standard approach. While we do not perform the integrals by simple quadrature (we still use Eq. (8)), it is clear that numerical integration would no longer seriously affect the results. The determination of the influence coefficients is still simpler, since special cases are not required (as when integration passes through a singular point of the kernel). Now, however, one must decide where to locate the kernel singularities.

We use the two strategies for locating the kernel singularities outside the contour. The first strategy places $z_{k}$ on the perpendicular bisector of the straight line between adjacent nodes at a distance $f$ times the local node spacing, $\left|z_{k}-z_{k-1}\right|$, away from the straight line. This strategy has two potential difficulties. If $f$ is too small, the singular point may lie inside an actual convex-curved contour. If $f$ is too large, the singular point may lie inside the domain of a highly contorted contour. The second strategy places all singular points on a circle whose center is roughly at the centroid of the contour. This strategy avoids the difficulties of the first, but it may not place the kernel singularities sufficiently close to the contour to capture nearly singular behavior of the solution. The second strategy also has the freedom of placing any number of kernel singularities on the circle, and hence, the algebraic system can become more or less overdetermined.

Figure 15 shows the error computed using the first strategy with two different values of $f$; in this case the nodes are placed evenly on a circular contour with $\beta=\sin z$. Here, we see that the convergence is exponential and that the overdetermined system is again the best. Figure 15 indicates that placing the singularities

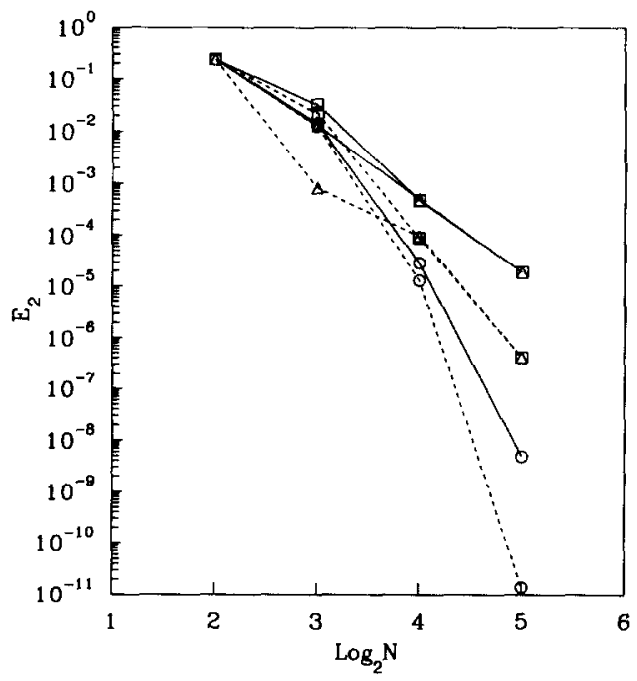

FIG. 15. Convergence for desingularized boundary integral method (circular contour, inner problem, $\beta=\sin (z), \phi$ is given): squares-weak system; triangles—strong system; circles-overdetermined system; dashed line $-f=1$; solid line $-f=2$. 


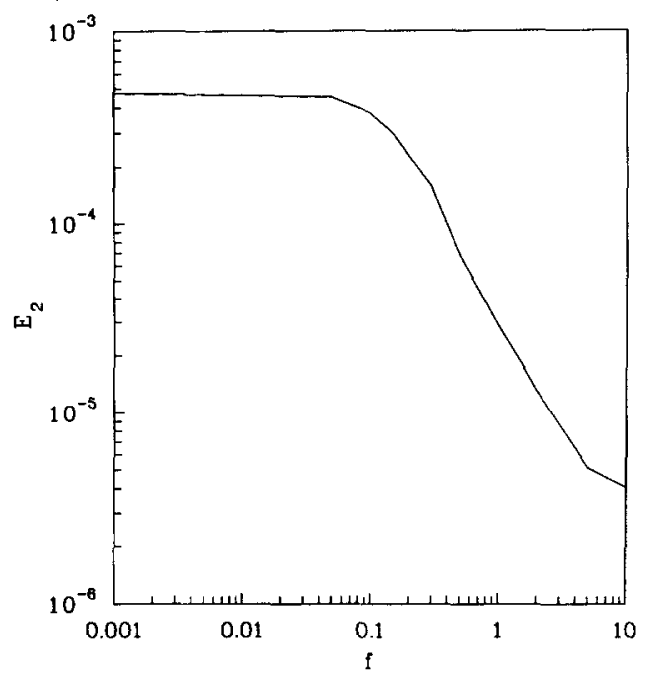

Fig. 16. Effect of desingularization parameter on error (circular contour, inner problem, $N=16$, $\beta=\sin (z), \phi$ is given $)$.

further from the contour is preferable. Figure 16 shows this to be the case for the same example with fixed $N=16$. However, a price is paid in that the algebraic system becomes less diagonally dominant as $f$ increases, requiring more iterations and a tighter tolerance on the convergence parameter to achieve the acquired accuracy. For this contour we note that when $f<(1-\cos (\pi / N)) / \sin (\pi / N) \approx 0.05$ for $N=16$, the singular point actually moves inside the contour. Figure 16 shows this happens without greatly changing the error.

Figure 17 also shows exponential convergence for the circular contour when the solution is singular outside the domain, as before $\beta=1 /(z-(1+\varepsilon))$. In this case, a semi-log plot indicates the true exponential character of the convergence. Only the overdetermined results are shown since the weak and strong system results are nearly as good. The nodal spacing must be small compared to $\varepsilon$ for exponential convergence to be achieved. As a result, the convergence when $\varepsilon=0.1$ (not shown) is very poor all the way to $N=64$. As might be expected, the most accurate solutions are no longer obtained when $f$ is large, since placing the kernel singularities further away cannot capture the effect of solution singularities close to the contour.

For noncircular contours, Fig. 18 shows that the overdetermined system still has smaller errors, although not significantly smaller than those for the desingularized solution of the strong or weak system. As before for noncircular contours, all systems have second-order convergence. (The weak system is not shown since its results are very similar to the strong system.) While the desingularized method greatly improves the accuracy of the weak and strong system, it only slightly improves the accuracy of the overdetermined system.

For the examples in Fig. 15-18, the desingularized method is better than the 


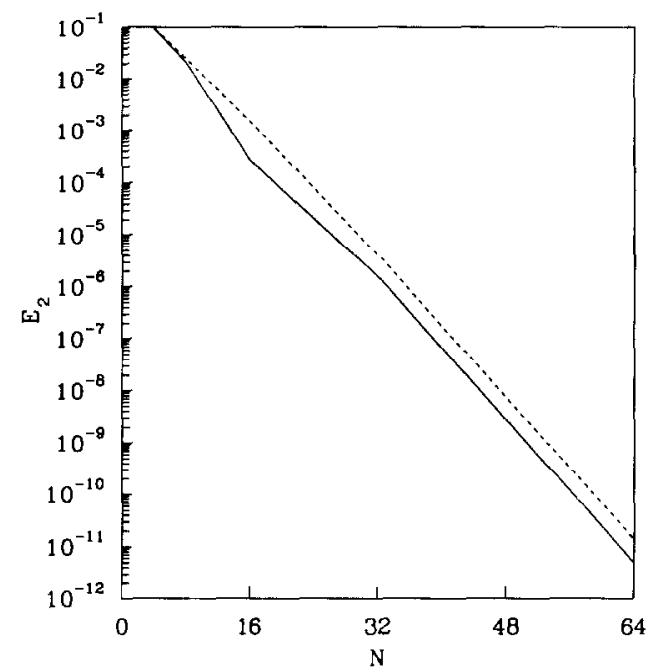

Fig. 17. Convergence of desingularized method for nearly singular solution (circular contour, inner problem, $\beta(z)=1 /\{z-(1+\varepsilon)\}, \varepsilon=1.0, \phi$ is given $)$. Only overdetermined results are shown since other system errors are nearly identical: dashed line $-f=1$; solid line $-f=3$.

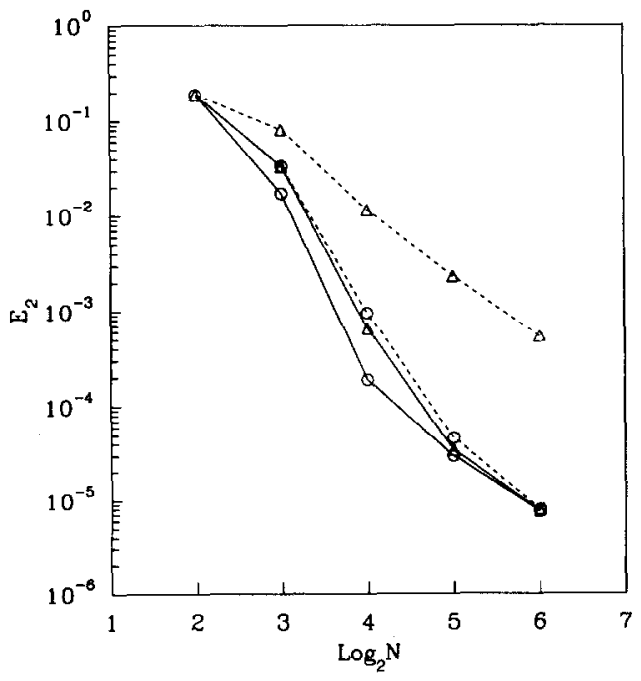

FIG. 18. Convergence comparison for elliptical contours (ellipse, $e=0.436$, inner problem, $\beta=\sin (z)$, $\phi$ is given): triangles--strong system; circles-overdetermined system; dashed line-standard method; solid line-desingularized method, $f=1$. Weak system results are nearly identical to those of the strong system. 
TABLE IV

$E_{2}$ for various $N_{s}(N=16)$

\begin{tabular}{rcc}
\hline$N_{s}$ & Combined weak and strong & Strong \\
\hline 8 & -3.373 & - \\
12 & -4.217 & - \\
15 & 4.677 & - \\
16 & -4.677 & -3.600 \\
17 & -4.677 & -3.373 \\
31 & - & -4.043 \\
32 & -4.678 & -4.140 \\
33 & - & -4.090 \\
34 & - & -4.127 \\
64 & - & -4.007 \\
\hline
\end{tabular}

method of Greenhow et al. However, both strategies of placing the kernel singularities off the contour do not yield quite as accurate results as the standard approach when the contour has a corner. The convergence rate is still the same and the absolute errors increase slightly (not shown but typically $25 \%$ ). Further work is required to determine how to place the singular points next to the contour corners; however, our computations show that decreasing $f$ or $R_{s}$ near the corners is not beneficial, even when no solution singularity exists there.

The second strategy of desingularization allows an arbitrary number of kernel singularities to be used in formulating the algebraic system. When the real and imaginary systems are used, the number of singular points, $N_{s}$, can be less than the number of nodes, $N$, actually $N_{s} \geqslant N / 2$. Table IV shows typical error results using variable $N_{s}$. All results are for a unit circle contour, $N=16$, and $\beta=\sin z$, with $N_{s}$ singular points equally spaced on a radius $R_{s}=1.5$. Table IV shows that $N_{s}$ can be reduced below $N$. Increasing $N_{s}$ further increases the accuracy insignificantly. However, increasing $N_{s}$ for the strong equations to overconstrain the system can be beneficial if $N_{s}$ is twice $N$. Table IV does not show that overdetermining the algebraic system in this manner is more expensive: more logarithms must be computed to form the influence the matrix and the resulting system is not as diagonally dominant (more iterations are required to solve the algebraic system).

\section{Conclusions}

Error analysis of complex variable boundary integral methods is made by truncation error analysis and numerical investigation. The overdetermined system is the most efficient and accurate in almost all cases by averaging errors of the opposite sign for the solutions of the strong and weak systems. The convergence rate of the overdetermined system is fourth-order for circular contours, while the other systems exhibit second-order convergence. When the contour is not circular, the convergence for all three systems is second-order, but the overdetermined system is 
significantly more accurate for moderate $N$. All systems have difficulties at contour corners, which can be alleviated by introducing a modified complex potential such that the derivatives are zero at the corner. The overdetermined system takes the fewest number of iterations in our conjugate gradient iteration procedure. As a result, the computational time is less than twice that of the weak or strong system. The increased accuracy of this method more than repays this additional cost.

For circular contours, exponential convergence can be achieved by kernel desingularization. The exponential convergence is destroyed when nodes are unevenly placed. The overdetermined system is, for the most part, the most efficient. This modification performs poorly when a contour corner is present.

The same approach may be extended to 3-dimensional potential problems by using two sets of integral equations, one of which is derived from Green's theorem and the other the normal derivative of Green's theorem. Hence, the advantages of solving an overdetermined system may yield greater efficiency and accuracy for 3-dimensional problems as well.

\section{ACKNOWLEDGMENTS}

This work was partially supported by Naval Research Laboratory Contract N00014-85-K-Z019, ONR Contract N00014-87-0509, and the Program in Ship Hydrodynamics at The University of Michigan, funded by the University Research Initiative of the Office of Naval Research Contract N000184-86-K0684. The authors acknowledge S. W. Joo and L. Pall for their helpful discussions and suggestions.

\section{REFERENCES}

1. M. Grfenhow, T. Vinje, P. Brevig, and J. Taylor, J. Fluid Mech. 118, 221 (1982).

2. C. Lal and T. V. Hromadka II, in Proceedings, ASCE Hydraulic Division Specialty Conference, Orlando, Florida, 1985 (unpublished).

3. J. W. Dold and D. H. Peregrine, "Steep Unsteady Water Waves: An Efficient Computational Scheme," School of Mathematics, Univ. of Bristol Report AM-84-04, 1984 (unpublished).

4. W. W. Schultz, in Proceedings, 11th IMACS World Congress, Vol. 2 (Oslo, Norway, 1985), p. 219.

5. J. L. Hess, Comput. Method Appl. Mech. Eng. 2, 1 (1973).

6. J. MoraN, An Introduction to Theoretical and Computational Aerodynamics (Wiley, New York, 1983).

7. M. A. Kelmanson, Comput. Fluids 11, 307 (1983).

8. G. R. Baker, D. I. Meiron, ANd S. A. OrszaG, J. Fluid Mech. 123, 477 (1982).

9. G. GoluB, Num. Math. 7, 206 (1965).

10. R. Fletcher and C. M. Reeves, Comput. J. 7, 149 (1964).

11. D. G. Dommскмuth AND D. K. Yue, in Proceedings, 16th ONR Symposium on Naval Hydrodynamics, Berkeley, California, 1986.

12. P. Grisvard, Elliptic Problems in Non Smooth Domains (Pitman, New York, 1985).

13. H. I. G. Pina, J. L. M. Fernandes, And C. A. Brebila, in Proceedings, 3rd Int. Seminar on B.E.M., Irvine, California, 1981.

14. W. M. LiN, J. N. Newman, ANd D. K. YuE, in Proceedings, 15th ONR Symposium on Naval Hydrodynamics, Hamburg, Germany, 1984.

15. C. Patterson ANd M. A. Sheikh, in Boundary Element Methods, edited by C. A. Brebbia (SpringerVerlag, New York/Berlin, 1981).

16. C. A. BrebBiA AND R. ButTERfield, Appl. Math. Modelling 2, 132 (1978). 